\section{L'INTERRUPTION SACRAMENTELLE DES RITES D'EXISTENCE}

Dans cette contribution ${ }^{1}$, j'entends démontrer que la réflexion théologique actuelle à propos des rites et des sacrements ne saurait méconnaître l'importance de l'analyse anthropologique. Mais, en même temps (et plus qu'autrefois), elle se doit de demeurer attentive à la spécificité chrétienne de la praxis sacramentelle. Je montrerai que ceci ne signifie nullement que la théologie se retire du dialogue interdisciplinaire (et interreligieux), bien au contraire. Pour ce faire, je partirai d'une lecture critique de l'article dans lequel Edward Schillebeeckx dessine les contours de sa nouvelle sacramentologie ${ }^{2}$. Ensuite, $j$ 'identifierai et développerai la problématisation de cet article à partir de la question de l'analyse du contexte actuel. Or, cette problématique sera comprise différemment selon que cette analyse sera perçue en termes de sécularité, ou plutôt en termes de pluralité. Une théologie qui part de la première analyse, comme la théologie moderne de la corrélation, semblera mettre l'accent principalement sur la continuité entre foi chrétienne et contexte, tandis que la théologie anti-moderne et, d'un point de vue chronologique, post-moderne, insistera sur la discontinuité absolue entre les deux. Une théologie qui s'appuie sur une analyse contextuelle comprise en termes de pluralité devra parfaire sa méthode, et sera capable de penser en même temps, dans le paradoxe de l'interruption, la continuité et la discontinuité.

1. Comment Schillebeeckx perçoit-il la problématique de la continuité et de la discontinuité dans le rapport entre rites d'existence et sacrements?

La fracture entre anthropologie et théologie a, selon Schillebeeckx, abouti à ce que la ritualité chrétienne soit coupée de l'existence. Par rapport à

1. Traduction par Christophe HERINCKX, aspirant au F.N.R.S., Belgique, associé à la faculté de théologie et de droit canonique, U.C. Louvain-la-Neuve.

2. E. SCHILLEBEECKX, Naar een herontdekking van de christelijke sacramenten. ritualisering van religieuze momenten in het alledaagse leven, dans Tijdschrift voor Theologie 40 (2000) 164-187. cela, il mentionne les représentations de foi tombées en désuétude comme étant la cause principale de la désaffection des gens par rapport à la pratique sacramentelle. Ils quittent l'Église parce que celle-ci ne parvient plus à ritualiser l'existence de manière adëquate. D'où le fait que, en élaborant son nouveau projet, Schillebeeckx se focalise sur l'aspect suivant: de quelle façon pouvons-nous comprendre les sacrements aujourd'hui, de telle façon qu'ils puissent avoir prise sur l'existence humaine? La solution consiste à concevoir les sacrements en termes de «ritualisation», en lien avec l'étude des rites et de la ritualité émanant des sciences humaines et naturelles (globalement décrites comme Ritual Studies). Ce point de vue se rapporte à une catégorie anthropologique renvoyant au fait qu'une praxis rituelle, aussi bien sur le plan religieux que sur le plan profane, est propre à l'être-homme, et est enracinée dans un jeu complexe de lois bio-psychiques, socio-biologiques, psychologiques, sociologiques, linguistiques et anthropologico-culturelles. De cette façon, Schillebeeckx accentue la nécessité anthropologico-structurelle d'une praxis sacramentelle chrétienne. C'est comme s'il voulait signifier à ceux qui s'en sont tenus éloignés qu'il est très humain de ritualiser l'existence, et que - si cette évaluation est correcte - l'être-chrétien inclut une participation à ces rites. Schillebeeckx espère, en particulier, pouvoir trouver les catégories qui permettront de repenser les sacrements comme des ritualisations de l'existence. C'est surtout le terme de performance qui, ici, a attiré son attention.

Schillebeeckx met donc avant tout l'accent sur la continuité fondamentale existant entre le besoin humain de ritualité, structurel et général, et la praxis sacramentelle, et, par conséquent, il met en garde contre une totale discontinuité.

Un mauvais côté chez les liturgistes et les théologiens est souvent qu'ils ne montrent aucun intérêt pour ce qu'est la ritualité 'en général', alors que celle$c i$, en tant que structure, est pourtant susceptible d'apporter une contribution à leur quête de la signification propre de leurs traditions rituelles particulières ${ }^{3}$.

Une analyse bienveillante, mais cependant critique, des résultats de la recherche anthropologique en cette matière est, à son avis, indispensable pour une compréhension correcte de ce dont il s'agit dans les sacrements, ou de ce dont il devrait s'agir. Le dialogue entre théologie et anthropologie est ainsi compris dans une corrélation critique réciproque ${ }^{4}$, qui offre à la théologie la possibilité de se renouveler en se recontextualisant, et donc de corriger la pratique sacramentelle de l'Église, là où c'est nécessaire. Pour autant que, à partir de ce qui est général, ce qui est

3. SCHILLEBEECKX, Naar een herontdekking, p. 174.

4. Ibid., pp. 175-176. 
spécifique puisse être compris et identifié. Bref, ce que Schillebeeckx accentue, c'est la continuité irréductible existant entre ce qui est humain et ce qui est chrétien. Cela se manifeste clairement lorsque Schillebeeckx explique plus avant son projet, et donne une description de ce que signifie théologiquement la praxis sacramentelle:

Dans les sacrements se cache toujours une double dimension, l'une anthropologique et l'autre 'théologale', orientée vers Dieu, qui fusionnent',

ce qui est explicité comme suit:

Pour les chrétiens, cet ensemble rituel est déjà, comme tel, la médiation divine de grâce - telle qu'elle se réalise dans et à travers sa propre puissance performative, au niveau cognitif, émotionnel et esthétique - de la performance animée par la foi chrétienne ${ }^{6}$.

À travers un traitement théologique de vues tirées des Ritual Studies, les sacrements peuvent, selon Schillebeeckx, être interprétés d'une façon nouvelle comme «une rencontre de Dieu existentialo-émotive». Schillebeeckx a assurément le mérite de montrer clairement que, dans les sacrements, la dimension théologique et l'aspect anthropologique vont de pair, que, d'un côté, en un sens humain général, ils obéissent aux lois de la ritualisation, et que, d'un autre côté, ils expriment la spécificité de la foi chrétienne et constituent un chemin vers la rencontre de Dieu, vers la connaissance de Dieu. Mais ce qui manque c'est, à mon avis, une problématisation approfondie de la manière dont la dimension rituelle et la dimension théologique s'accompagnent mutuellement (et une réflexion à ce propos), c'est-à-dire sur la façon dont ritualité et sactamentalité vont de pair - et précisément une telle réflexion est aujourd'hui nécessaire! Pour Schillebeeckx, les sacrements sont valables parce qu'ils sont d'authentiques rites d'existence. Je ne conteste pas cette donnée en ellemême, mais je me demande ce qui, pour les chrétiens, transforme les rites existentiels spécifiques, pratiqués dans les communautés de récit chrétiennes, en sacrements - et comment je peux concevoir cela de la façon la plus opportune dans notre contexte actuel.

\section{2. Éléments de problématisation}

Ma question ne porte donc pas directement sur la continuité structurelle existant entre la «ritualisation», comme catégorie anthropologique, et la praxis sacramentelle chrétienne. Par ailleurs, je n'entends en aucun cas contester le fait que la théologie ne peut que tirer profit de la recherche

5. SCHILLEBEECKX, Naar een herontdekking, p. 183 (je souligne).

6. Ibid., p. 184 (la mise en italique est de Schillebeeckx). interdisciplinaire. Mieux encore: cela fait partie du cœur même du concept de recontextualisation auquel j'adhère moi-même ${ }^{7}$. En outre, même ce que Schillebeeckx nomme la dimension théologale (la relation entre Dieu et homme/société/monde) ne peut être clarifié qu'à travers un dialogue interdisciplinaire avec la finalité théologique. C'est toujours en tant qu'êtres humains et à partir de notre environnement linguistique complexe que nous parlons de Dieu et à Dieu. Au contraire: c'est précisément à partir d'un tel échange interdisciplinaire, à partir de la rencontre entre foi chrétienne et contexte, que je me demande si la seule accentuation de la continuité entre ceux-ci constitue une stratégie théologique et pastorale fructueuse pour aujourd'hui.

Schillebeeckx fait partir sa réflexion - ainsi pourrait-on l'envisager de la «non-pratique rituelle et liturgique de fait» des catholiques «de la périphérie» (selon lui à cause des représentations de foi désuètes). Or, à mon sens, l'une des grandes difficultés, d'un point de vue pastoral, est que les sacrements et les autres rites chrétiens - particulièrement quand nombre de «catholiques périphériques» et de post-chrétiens y participent encore - ne fonctionnent plus qu'en tant que rites d'existence, leur dimension narrative chrétienne et théologique étant pour ainsi dire totalement occultée 8 . Ne fonctionne alors de facto que la dimension anthropologique, celle-ci n'étant plus fusionnée avec la dimension théologale: la rencontre avec le Dieu de Jésus-Christ dans l'imitation

7. Voir, entre autres, le premier chapitre de L. BoEve, Onderbroken traditie. Heeft het christelijk verhaal nog toekomst?, Kapellen, 1999.

8. Lorsque l'on demande aux gens de se définir eux-mêmes, $47,4 \%$ des Belges se considèrent comme catholiques, $1,2 \%$ se qualifient de protestants, et $15,3 \%$ se disent chrétiens, mais sans être protestants ou catholiques. Voir K. DOBBELAERE (coll.), Verloren zekerheid. De Belgen en hun waarden, overtuigingen en houdingen, Tielt, 2000. Cependant, les chiffres les plus parlants concernent bien sûr la participation de plus en plus faible aux célébrations eucharistiques, et de plus en plus, également, la baisse $\mathrm{du}$ nombre des baptêmes et des mariages célébrés à l'église: en 1967, 52 \% des Flamands (soit 42,9\% des Belges) allaient à l'église chaque semaine, alors que, en 1998, il 'n'y en avait plus que $12,7 \%$ (soit 11,2 \% des Belges); en 1967, 96,1 \% des enfants, en Flandre, étaient baptisés, en 1998 plus que $73 \%$. Le pourcentage concernant la Belgique est, pour l'année 1998, de $64,7 \%$, soit plus de $8 \%$ de moins qu'en Flandre. Les chiffres concernant la proportion de la population qui se marie encore à l'église sont: pour la Flandre, de $91,8 \%$ en 1967 , de $51,2 \%$ en 1998; pour la Belgique, de $86,1 \%$ en 1967 , de 49,2 \% en 1998 (cfr. ibid., p. 23). Par ailleurs, ces personnes participant aux rites de l'Église catholique ne sont pas toutes vraiment impliquées dans la vie de l'Église, tandis que des personnes non-membres de l'Église y participent également, du fait qu'il existe peu d'alternatives, en dehors de l'Église, permettant de célébrer les moments de passage importants de l'existence (cfr. ibid., pp. 117 et 136). Non seulement aussi bien les «membres centraux» que les «catholiques moyens» participent aux rites ecclésiaux lors des moments de transitions existentiaux, mais également $80 \%$ des membres périphériques de l'Église, et $45 \%$ des non-membres de l'Église ( $42 \%$ pour le baptême, $48 \%$ pour les funérailles). 
(dans la pratique et la réflexion, dans l'éthique et la ritualité). Autrement dit: ils fonctionnent de manière post-chrétienne. Notamment en raison du manque d'alternatives claires, beaucoup continuent de participer aux sacrements en vue de ritualiser l'existence, et non pas en vue de rencontrer Dieu. Beaucoup ne sont pas seulement (ou pas d'abord) devenus étrangers au récit chrétien de foi et à la pratique chrétienne de foi à cause d'un imaginaire vieilli, mais également (et avant tout) à cause du processus socio-culturel de détraditionalisation. À une époque d'individualisation, les chrétiens ne deviennent résolument chrétiens que s'ils en font eux-mêmes le choix; ils ne le sont plus «de naissance». Vus dans la seule perspective des rites existentiels, les sacrements ne sont en principe rien de plus que des rites disponibles sur le marché des conceptions de la vie, d'où les gens peuvent, s'ils veulent ritualiser leur existence, tirer des éléments ${ }^{9}$.

Dans ce cas, d'un point de vue pastoral, l'insistance sur la seule continuité fonctionne de manière contre-productive. Naturellement, les sacrements chrétiens ont trait, en définitive - d'un point de vue théologique - à la rencontre avec Dieu: la célébration de l'existence à travers le rite et le sacrement situe cette existence, avec ses joies et ses peines, avec le quotidien et le non-quotidien, dans la relation au Dieu révélé en JésusChrist, et dans la confession toujours renouvelée de ce que le mystère de cette vie, de la réalité, se nomme Amour.

Lorsque les rites d'existence chrétiens ne concernent plus la dimension narrative chrétienne et théologique, alors, d'un point de vue théologique, ils sont trop aisément fonctionnalisés, ils sont réduits à la fonction qu'ils remplissent en faveur de l'existence et de la coexistence humaine. Les hommes ont besoin de ritualité - d'où l'on peut conclure que, s'ils n'y sont pas attentifs, ils nient leurs racines bio-psychologique et sociobiologique, et mettent ainsi en danger leur existence psychologique et sociale. Dans cette perspective, la ritualisation peut même se muer en thérapie. Il est vrai que la ritualisation est indispensable pour un équilibre psychologique et social, et qu'elle agit même de manière thérapeutique, mais l'inverse n'est pas automatiquement vrai: celui qui considère les rites seulement de cette manière sape leur efficacité et ne peut plus aucunement accéder à leur dimension théologale. L'étude de la ritualité par la philosophie anthropologique et les sciences humaines nous apprend également que les rites existent par leur «incarnation» particulière. Certains développent cette piste et parlent de l'ancrage dans l'espace

9. Une partie de ce que Karel Dobbelaere a appelé le «catholicisme à la carte» doit assurément être élucidée à partir de cette façon de voir. Cfr. K. DOBBELAERE, Het «volkgods" de mist in? Over de kerk in België. Kerk-zijn in de huidige wereld - 1 . Sociologische benadering (Nikè: Didachè), Leuven; 1988. symbolique, espace dans lequel l'homme vit dans le rite et la tradition, dont l'homme lui-même ne dispose pas. Celui qui entend malgré tout en disposer à son gré court le risque de détruire la source instituante de signification $^{10}$. D'un point de vue théologique et méthodologique, cette optique représente également une fonctionalisation de la pratique sacramentelle chrétienne, car, ici aussi, cette pratique est légitimée exclusivement à partir de la donnée anthropologique selon laquelle la donation humaine de sens est «incarnée» dans des rites et des pratiques prédonnés.

Une telle fonctionalisation anthropologique de la pratique rituelle chrétienne se manifeste ainsi sous plusieurs formes, aussi bien dans les cercles chrétiens que non-chrétiens. C'est ce qui se passe chez ceux que l'on désigne par le terme de progressistes: «nous devons créer des rites, parce que nous n'en avons pas (ou bien ils ne sont plus valables)»; et chez les conservateurs: «les rites sont des réalités dont on ne peut disposer, auxquelles nous devons nous soumettre». Ces différentes positions se fondent exclusivement sur la nécessité anthropologique des rites, et situent, chacune à sa manière, la praxis sacramentelle chrétienne à l'intérieur de cette nécessité. Dans les deux cas, les rites se muent en pratiques fonctionnant dans le cadre d'une perspective humaine générale, à l'intérieur de «la» structure anthropologique de «l'homme». La narrativité chrétienne ne représente alors plus qu'un remplissage particulier; elle fournit le cadre narratif.

On ne peut aucunement reprocher à Schillebeeckx de fonctionnaliser la praxis sacramentelle. Il tient assurément compte de leur dimension théologale, et développe celle-ci à partir de ses recherches christologiques. Par contre, ce que je me demande, c'est si sa méthode théologique n'agit pas de manière contre-productive. Son argumentation selon laquelle les sacrements se situent dans le prolongement de notre êtrehomme n'en vient-elle pas à occulter ce qu'il entend dire par ailleurs, à savoir que, à travers cette ritualisation humaine, les chrétiens rencontrent Dieu? Pour beaucoup, cette dimension théologale apparaîtra comme un simple redoublement ou une simple coloration narratifs de ce qui se passe déjà au niveau humain. Ainsi, par exemple, dans le cadre de la pastorale des rites d'existence, on se demande parfois pourquoi les textes

10. Voir, à ce propos, les réflexions de Herman DE DIN, entre autres, dans Hoe overleven we de vrijheid? Modernisme, postmodernisme en het mystiek lichaam, Kapellen, 1993, et de Paul MOYAERT, entre autres, De mateloosheid van het christendom. Over naastenliefde, betekenisincarnatie en mystieke liefde, Nijmegen, 1998; pour des études critiques sur ces auteurs, voir respectivement: L. BOEvE, Hoe overleven we de kloof tussen kennis en leven? Een theoloog over het uitdagende denken van Herman De Dijn, dans Tijdschrift voor Theologie 36 (1996) 221-245; S. VAN DEN BossCHE, Geloven in surrealistisch perspectief? Een theologisch wederwoord bij de symbool- en sacramententheorie van Paul Moyaert, dans Bijdragen 60 (1999) 324-345. 
liturgiques doivent «toujours» nécessairement parler de Dieu ou être tirés de la bible, et si l'on ne pourrait pas tout aussi bien emprunter à d'autres traditions.

Dans le même sens, notre principale interrogation est la suivante, en rapport à la communication que fit Paul Post à Soesterberg ${ }^{11}$. Il est en effet remarquable que, dans la perspective liturgico-rituelle que développe Paul Post, cette dimension théologale n'est même pas mentionnée, donc encore moins élaborée, tandis qu'elle apparaît par contre dans «la présentation du projet de recherche défendu (par lui)». Par rapport à cela, dit-il, «on peut désormais estimer que la théologie de la liturgie, qui contient de plus en plus aussi une théologie sacramentelle, qui faisait traditionnellement partie de la théologie systématique classique, est liée à des voies d'analyse liturgico-rituelle» ${ }^{12}$. Cela dit, où se produit cette relation? Comment se réalise-t-elle? Comment, en tant que théologiens, pouvonsnous montrer qu'il y a plus ici qu'un redoublement? Par ailleurs, je me suis demandé à plusieurs reprises si, dans ce discours, le mot «liturgie» s'est transformé en un terme purement descriptif de la pratique rituelle des chrétiens, ou s'il comprend encore, malgré tout, la dimension théologale ${ }^{13}$. Et si ce lien n'a pas été fait jusqu'à présent, est-ce parce que cela doit encore être effectué?; ou ce lien ne doit-il justement plus être posé, parce que tout aurait déjà été dit, d'une point de vue liturgicorituel? En d'autres termes: quelle est la réponse à la question de départ à propos de la continuité et de la discontinuité entre les rites d'existence et les sacrements?

11. C'est précisément en rapport à ce point que Post a apporté sa contribution à ce volume, et fait ressortir la finalité théologique. Une question demeure toutefois, à savoir si la suspension et la relativisation méthodiques de la théologie n'aboutit pas finalement à une incapacité d'accéder à la dimension théologale. Voir, en rapport à cela, ce que j'écris plus loin au sujet de la théologie moderne de la corrélation.

12. Voir, dans ce recueil: P. POST, Life Cycle Rituals: A Ritual-Liturgical Perspective, p. 12 (l'accentuation est la mienne).

13. Cette impression est renforcée du fait que Post, dans son intervention à Soesterberg, situe sa propre approche ethnologique dans ce qu'il désigne comme étant le troisième secteur des Ritual Studies: «L'étude de la liturgie est ici principalement considérée comme une étude anthropologique et rituelle», à distinguer (à moins qu'il ne le faille justement pas?) du quatrième secteur, qui prolone cette perspective et amène très explicitement les Ritual Studies dans le giron de l'étude liturgique, entendue au sens d'un projet théologique, et tente d'insérer les résultats des chercheurs alliés des Ritual Studies dans une théologie de la liturgie». La version figurant dans le présent recueil ne reprend pas le passage concerné; Post a également présenté cette analyse dans: Personen en patronen: Literatuurbericht liturgiewetenschap, dans Praktische theologie 48 (2001) 89-90.
3. La sécularité (l'«humain-en-général») dans une perspective de pluralité

Dans ce qui suit, je vais tenter de montrer que, en raison du changement de contexte, la méthode moderne de corrélation ${ }^{14}$ pratiquée par Schillebeeckx, et de nombreux théologiens de sa génération, ne fonctionne plus de manière satisfaisante. La théologie y est considérée comme le fruit de la rencontre réciproque critique (de la co-relation) entre la tradition chrétienne d'une part et la culture et la société modernes séculières, en train de s'installer, d'autre part. Cela ne signifie pas que la rencontre entre tradition et contexte ne fonctionnerait plus comme moteur pour une recontextualisation, mais que la façon d'aborder méthodiquement cette rencontre doit être réformée de manière post-moderne. Les conséquences de cette nécessité pour la méthodologie théologique seront traitées dans un point suivant. Pour l'instant, nous nous intéressons à la façon d'analyser le contexte actuel.

À l'aide de quelles catégories interprétons-nous le contexte actuel? La théologie la plus moderne comme la plus anti-moderne - donc émanant à la fois d'auteurs tels que Küng, Rahner et Schillebeeckx, et de von Balthasar et Ratzinger - part du principe selon lequel un processus de sécularisation a amené une cassure dans la synthèse pré-moderne entre christianisme et culture, religion et monde. L'homme a découvert la puissance de la raison, de la science et de la technique modernes et, à partir d'une conscience subjective, a initié des mouvements d'émancipation. Il s'agit

14. Dans Tussentijds verhaal, Bruges, 1975, pp. 60-61, entre autres, Schillebeeckx décrit cette théorie critique de la corrélation, laquelle est constituée de trois étapes: premièrement, la recherche de structures constantes de l'expérience chrétienne fondamentale, dans le Nouveau Testament; deuxièmement, l'analyse du monde contemporain des expériences, aussi bien en un sens culturel général que spécifiquement chrétien; et troisièmement, la corrélation critique ou la confrontation des deux aspects (qui sont la source de la théologie). Plus tard, Schillebeeckx préférera décrire la «corrélation» comme une «interrelation» (cfr. Theologisch geloofsverstaan anno 1983, Baarn, 1983, p. 9). À la page 10 de cet ouvrage, Schillebeeckx affirme même que le terme «corrélation» prête à confusion. La théologisation authentique, écrit-il, s'accomplit «à travers deux phases, qui forment conjointement un ensemble dialectique. [...] Nous ne comprenons en effet la tradition chrétienne qu’à partir des questions qui nous sont transmises à travers la situation actuelle dans laquelle nous vivons; la compréhension du passé implique déjà une interprétation du présent. Et inversement, notre compréhension du présent subit elle-même l'influence historique de la tradition chrétienne». Voir également déjà Gerechtigheid en liefde, Bruges, 1977, p. 63: «La théologie chrétienne concerne précisément une interrelation entre, d'une part, une "Gegenwartsanalyse» et, d'autre part, une analyse de et une méditation herméneutique sur l'expérience historique de la vie chrétienne, dans le but de tirer une orientation de cet ensemble, orientation dans laquelle nous pouvons, en tant que chrétiens, vivre d'une façon responsable en vue de l'avenirs. Examinée de plus près, cette théorie de l'«inter-relation» demeure en fait attachée aux trois étapes mentionnées plus haut. 
de l'homme qui a appris à réfléchir sur sa situation, sur l'existence et la coexistence, dans un discours et une donation de sens séculiers, à propos des nombreux domaines de son existence et de la coexistence (lesquels se sont détachés du milieu traditionnel, à travers une différenciation fonctionnelle). C'est cet homme qui, dans la mesure où il est resté croyant car il devenait également possible de ne plus l'être -, a dû mettre en relation cette connaissance et cette donation de sens avec sa foi. Par rapport à ces données, les auteurs modernes et anti-modernes s'opposent surtout sur la question de savoir jusqu'à quel point l'autonomie de la sécularité peut être respectée théologiquement. Pour les théologiens modernes, la modernité représentait un processus d'apprentissage avec lequel les chrétiens pouvaient (ou devaient) entrer en dialogue de manière fructueuse; pour les théologiens anti-modernes, la modernité représentait une aliénation et de la prétention humaine qui, l'une et l'autre, devaient être vaincues.

Cependant, la thèse de la sécularisation est fortement remise en question. Deux anciens protagonistes de cette thèse, Harvey Cox et Peter Berger, ont pris leurs distances par rapport à cette dernière et signalent une renaissance religieuse globale ${ }^{15}$. Le thèse de la sécularisation ellemême, associée à la «théorie de la somme zéro», affirmait que la modernisation est un processus continu devant bouter progressivement la religion hors de la société et de la culture; la somme de la modernisation et de la religion serait ainsi toujours nulle: plus il y aurait de religion, moins il y aurait de modernisation; et surtout l'inverse: plus il y aurait de modernisation, moins il y aurait de religion. Or, la religion a survécu à la modernisation, même en des lieux hyper-modernisés ${ }^{16}$. Aussi bien Schillebeeckx que Post semblent se référer tant soit peut à la thèse de la «désécularisation». Schillebeeckx met en doute le fait que la sécularisation serait la seule cause de l'abandon de la praxis sacramentelle par les catholiques de la périphérie (le problème étant, comme on l'a déjà dit, l'attachement ecclésial à des représentations de foi désuètes) ${ }^{17}$. Post, de son côté, se pose des questions nuancées au sujet de la théorie du désenchantement de la société, et a parlé d'une «effervescence de la religion et de la sacralité» à notre époque ${ }^{18}$. À cela, $j$ 'ajoute volontiers deux remarques. (1) Berger, de façon remarquable, écrit qu'il y a deux exceptions à la «thèse de la désécularisation». D'un côté, il y a ceux qui

15. Cfr. H. Cox, The Myth of the Twentieth Century. A Theological Overview, New York, 1999; P. BERGER, The Desecularisation of the World: A Global Overview, dans ID. (éd.), The Desecularisation of the World. Resurgent Religion and World Politics, Washington, DC, 1999.

16. Cox, The Myth of the Twentieth Century, pp. 135-136.

17. SCHLLLBEECKX, Naar een herontdekking, p. 165.

18. Post, Life Cycle Rituals, p. 20. sont supérieurement instruits, avec une formation occidentale, une sorte de sous-culture internationale, et, de l'autre côté, l'Europe occidentale, bien que la situation y soit ambiguë. On y observe une importante défection par rapport à l'Église, d'abord dans les pays d'Europe du nord, et, depuis quelques temps, également dans le sud. Berger parle de la genèse d'une «Euro-culture séculière massive». Pourtant, s'appuyant sur des sociologues européens de la religion, il se demande si le terme de sécularisation est propre à décrire ces développements. Car «un ensemble de données indique de fortes survivances religieuses, dont la plupart sont généralement de nature chrétienne, malgré la désaffection étendue par rapport aux Églises organisées. Dans ce cas, un changement dans la situation institutionnelle serait une description plus correcte de la situation européennes ${ }^{19}$. (2) Deuxièmement, en lien avec cela, que le processus puisse encore être appelé sécularisation, ou qu'il faille parler de changement dans la situation institutionnelle (ou encore des deux à la fois), on peut assurément parler à bon droit de détraditionalisation et d'individualisation, accompagnées d'une pluralisation grandissante.

La mise en doute de la thèse de la sécularisation n'implique aucunement que les personnes qui, à cause de la modernisation, ont été emportées loin du récit chrétien, mais découvrent qu'ils sont «incurablement religieux et rituels», reviendront vers le christianisme. Même pas au cas où celui-ci parviendrait à renouveler, d'une manière adaptée, le langage de la foi (ce qui ne peut certainement pas servir d'argument pour ne pas accomplir cette tâche); il me paraît inintelligent - cela donnerait précisément encore plus de crédit à la thèse de la sécularisation - de situer la renaissance religieuse, y compris le «murmure religieux», sur l'axe «modernité-christianisme», mais alors à nouveau plus près du pôle «christianisme». Une perspective de pluralité rend mieux justice à cette renaissance multiforme et à la multiplicité religieuse, laquelle nous parvient par le biais de la mobilité géographique et mentale (c'est-à-dire respectivement par la migration et par les média). Il y a, de manière individuelle, interpersonnelle et collective, de multiples façons d'étudier, d'analyser, de vivre l'existence et la réalité, et de leur donner sens - et la façon théologale chrétienne est l'une d'entre elles (avec ses propres variétés).

Les sciences humaines et naturelles (occidentales), méthodiquement athées, ont une place inaliénable, mais spécifique, parmi les multiples manières de considérer l'existence et la réalité. Mais la sécularité, dans le cadre de laquelle il faut situer ces sciences, et qui sont structurellement liées à la culture et à la société occidentales (et, à travers la globalisation,

19. BERGER, The Desecularisation of the World, p. 10. Il poursuit: «Malgré tout, l'Europe demeure dans une situation très différente de celles d'autres parties du monde, et certainement de celle des États-Unis». 
à la culture et à la société mondiales), ne détermine plus à elle seule la dynamique du contexte auquel le récit chrétien se rapporte. En outre, par le biais de la critique émanant de la pensée post-moderne, la position soidisant neutre de l'observateur, occupée classiquement par la science et la rationalité, est fondamentalement mise en question, et les cercles herméneutiques à l'intérieur desquels opèrent ces dernières sont mis à nu. Néanmoins, au milieu d'une multiplicité de modèles et de théories, et, surtout, légitimées par leur effectivité et leur performativité, la science et la rationalité demeurent constitutives de la façon dont les Occidentaux, y compris les chrétiens entre eux, se situent dans l'existence ${ }^{20}$. D'où le fait que (dans leur développement ${ }^{21}$ ) elles conservent toute leur importance pour une théologie qui se recontextualise. Mais, comme il a été dit, le contexte ne doit plus être interprété uniquement en termes de sécularisation, et le rôle des sciences ne doit plus être interprété à partir de la thèse de la sécularisation

Précisément du fait que le contexte sera compris de manière plus exacte comme pluralité que comme sécularité, et que le récit chrétien se trouve situé à l'intérieur de cette pluralité comme un récit parmi d'autres, la question théologique la plus prégnante ne consistera plus à se demander comment la tradition chrétienne peut se rapporter de façon fructueuse à la sécularité moderne, débouchant sur un mode d'existence christiano-moderne significatif et plausible. La question consistera bien plutôt à se demander de quelle manière le récit chrétien se rapporte à la multiplicité. À ce niveau, on peut parler, de façon sensée, d'une perspective interne et d'une perspective externe, avec d'une part, ad extra, la découverte de sa propre particularité, et d'autre part, ad intra, l'examen de ce que signifie le défi du nouveau contexte pluriel, à travers la confrontation avec d'autres particularités, pour le développement de cette narrativité particulière. Dans la théologie moderne, ces deux dimensions sont à peine distinguées; à plus forte raison, elles ne sont pas pensées comme essentielles. Le mouvement ad intra est le (ou: est identique au)

20. Le sociologue de la culture Rudi Laermans indique, en lien à cela, que la modernisation s'est faite de pair avec une proto-professionalisation du milieu de vie; la connaissance scientifique vulgarisée est absorbée, ce qui conduit à une médicalisation, une juridisation, une psychologisation, etc., du milieu de vie. Cfr. R. LAERMANS, In de greep van de Moderne Tijd: Modernisering en verzuiling, individualisering na het naoorlogse publieke discours van de $A C W$-vormingsorganisaties. Een proeve van culuurocio gische duiding (K.U.L. Faculté des Sciences sociales. Département de Sociologie), Leuven, 1992, p. 10.

21. Voir, en rapport à cela, la façon dont Schillebeeckx renvoie à l'évolution d'une anthropologie statique vers une anthropologie dynamique du rite et de la ritualisation. Dans le cadre de la première, les rites sont considérés comme «non-régénérables», mais issus d'un "passé obscun» et inaccessible, de sorte que la créativité rituelle apparaît comme une contradiction en soi (Cfr. SCHILlEBEECKX, Naar een herontdekking, p. 169). mouvement ad extra: un christianisme qui se laisse interroger et enrichir par la culture séculière, et qui, ainsi, veut devenir crédible et significatif à l'intérieur de la culture séculière. Exprimé dans des catégories modernes, cela signifie que la particularité du christianisme est formulée de telle manière qu'elle apparaît comme parfaitement acceptable, si ce n'est comme une expression par excellence de l'homme, de la culture et de la société modernes ${ }^{22}$. En outre, certainement en ce qui concerne la Flandre et de grandes parties de l'Europe occidentale, la pluralisation a été longtemps ralentie en raison de la position largement majoritaire qu'y occupaient encore les Églises chrétiennes. D'un point de vue culturel et social (entre autres par le biais de la naissance des «piliers»), un lien s'est longtemps maintenu entre la culture moderne et la foi chrétienne.

Il est à noter que, tout ce qui a été dit ci-dessus, est le résultat d'un dialogue interdisciplinaire avec la philosophie et les sciences humaines, avec la finalité théologique ${ }^{23}$.

\section{Une perspective stricte de discontinuité n'offre pas de solution non plus}

Penser dans les termes de cette continuité est propre à une certaine façon de théologiser, née de la confrontation avec la modernité. La perte de plausibilité des présomptions modernes quant à la rationalité et à la construction de la société, c'est-à-dire des dits grands récits modernes, a fourni de nouveaux arguments aux critiques de cette manière de théologiser. Des théologiens anti-modernes ont vu ainsi leur position confirmée: l'«autonomie absolutisée» du sujet et du monde est, en ce qui les concerne, apparue comme une fiction, qui a finalement causé leur perte. Pour cela, quelques-uns parmi eux plaident en faveur d'une théologie vraiment post-moderne, en un sens chronologique: une théologie d'après la modernité, abandonnant celle-ci, ou du moins ses présupposés

22. Comme je l'ai écrit dans la description de la problématisation du symposium: "L'aspiration moderne à la liberté humaine et à la libération sociale devint un lieu de recontextualisation de la foi chrétienne en un Dieu qui se préoccupe du salut de l'homme. De là où la dignité de l'homme était respectée, Dieu ne pouvait être absent. Cette théologie partait du présupposé principiel selon lequel l'on pouvait constater une continuité entre modernité et foi chrétienne; il s'agissait d'établir une corrélation entre le message de salut de la foi chrétienne et le contexte moderne: 'la foi et l'existence (moderne) se trouvent dans le prolongement l'une de l'autre'. la théologie de la corrélation cherchait ainsi à établir un concensus entre la culture et la foi: le chrétien était pour le moins aussi moderne que n'importe quel homme moderne. Le projet moderne était un projet derrière lequel les chrétiens pouvaient se ranger avec les autres 'hommes de bonne volonté'».

23. Voir, à ce sujet, l'étude Onderbroken traditie, avec, par exemple, Critical Consciousness in the Postmodern Condition. A New Opportunity for Theology? dans Philosophy and Theology 10 (1997) 449-468. 
séculiers. Ils interprètent la crise post-moderne de la modernité (désignée par d'autres comme une radicalisation ou même la réalisation de la modernité ${ }^{24}$ ) comme la faillite de cette dernière. Pour cette raison, ils rejettent l'athéisme méthodique moderne comme étant sans avenir, et présentent des schèmes de pensée réclamant une relation plus originelle à Dieu comme point de départ de toute réflexion sur l'homme et le monde.

À ce propos, je renvoie à deux courants, qui déploient de telles stratégies indépendamment l'un de l'autre, tant du point de vue du contenu que de la méthode: le mouvement de la «Radical Orthodoxy», qui a d'emblée conquis une place intéressante dans le monde anglosaxon, et sans doute également quelques représentants du dit tournant théologique dans la phénoménologie française récente ${ }^{25}$.

Le mouvement de la «Radical Orthodoxy» s'appuie sur la critique post-moderne de la modernité dans la seule mesure où elle permet de «réclamer le monde à la théologie» ${ }^{26}$. En effet, la valeur de la pensée post-moderne réside, selon ces penseurs, dans le fait qu'elle démontre la faillite de la modernité séculière, laquelle débouche sur l'angoisse, en raison de la perte inhérente de sens et de valeur. «Ce qui émerge, c'est un projet théologique contemporain, rendu possible par la superficialité auto-consciente du sécularisme actuel». Aussi, ce groupe ne fait plus confiance au projet des théologies modernes de la corrélation, qui recherchent pour ainsi dire un lien interne avec «des expressions universelles de valeur immanente». Il critique en même temps un «baptême (théologique post-moderne) du nihilisme au nom de 'théologies négatives' mal comprises. Face à la transmission séculière de la vérité, celles-ci cherchent bien plutôt à reconfigurer la vérité théologique» $^{27}$. D'après ces penseurs, le recours à un cadre de pensée néoaugustinien permet de résoudre les apories modernes qui sont apparues dans le nihilisme post-moderne. La clé principale permettant d'effectuer cela est la catégorie de la «participation». Le fini ne conserve son intégrité, et même sa «mondanéité» que lorsqu'il participe à sa source

24. D'autres encore, tels Anthony Giddens et Ulrich Beck, parlent pour cette raison de «modernité réflexive».

25. Voir également, à ce propos: L. BoEve, Method in Postmodern Theology. A Case Study, dans L. BOEVE - J. RIES (éds.), The Presence of Transcendence: Thinking «Sacraments in a Postmodern Age (Annua Nuntia Lovaniensa, 42), Leuven, 2001, pp. 19-39.

26. Pour une esquisse de ce en faveur de quoi lutte le mouvement de la «Radical Orthodoxy», voir l'introduction de J. MILBANK - C. PICKSTOCK - G. WARD (éds.): Radical Orthodoxy: A New Theology, London/New York, 1999; et également J. MILBANK, The Program of Radical Orthodoxy, dans L.P. HEMMING (éd.), Radical Orthodoxy?-A Catholic Enquiry, Aldershot, 2000, pp. 33-45.

27. Introduction, dans MILBANK, et al. (éds.), Radical Orthodoxy, p. 15. infinie et éternelle, tandis que, dans le cadre de l'épistémologie moderne et du nihilisme post-moderne, cette intégrité finit par s'évanouir ${ }^{28}$.

Il vaut certainement la peine d'examiner si l'élargissement de la phénoménologie française vers ce que Janicaud a appelé «le tournant théologique» ne peut être interprété dans le même sens, bien que sa méthode de travail soit différente. Il semble en effet que, pour un certain nombre de ces auteurs, l'intégration de la philosophie dans une configuration théologique fournisse le critère de la bonne philosophie $e^{29}$. Par rapport à cette perspective, deux auteurs importants sont Jean-Luc Marion $^{30}$ et Jean-Yves Lacoste ${ }^{31}$. Les deux analysent la reproduction phénoménologique heideggerienne du monde comme fermeture. Le «monde» constitue, selon eux, un horizon de signification sans Dieu. Les logiques de la donation (Marion) et de l'attitude liturgique (Lacoste) font éclater cet horizon fermé, et manifestent la postériorité de celui-ci. Le monde - étants et Être - est toujours déjà donné (Marion); son caractère fermé est toujours déjà radicalement ouvert à partir de la rencontre avec 1'Absolu (Lacoste). Cela dit, Marion (dans une moindre mesure Lacoste) nie que sa méthode reposerait ultimement sur des bases théologiques. Au

28. Dans une critique adressée à Graham Ward, prononcée lors du deuxième congrès LEST, K.U. Leuven, Kevin Hart fait valoir que Ward et ses collègues attribuent de façon trop rapide un nihilisme à la modernité, nihilisme que les théologiens se doivent de dépasser. Ils oublient que, à l'intérieur de la théologie chrétienne elle-même, un nihilisme est à l'œuvre, qui est constitutif, et ils se laissent enfermer trop rapidement dans un grand récit chrétien, de type néoplatonicien et augustinien. Au lieu d'être une participation «harmonieuse», la foi chrétienne, selon Hart, se rapporte plus à une «implication dans», une «appartenance à» un Christ qui interrompt nos vies. Celui qui regarde les choses dans une telle perspective pourra peut-être découvrir la "déconstruction» au cœur du christianisme lui-même. Hart développe ceci dans The Trespass of the Sign: Deconstruction, Theology and Philosophy, Cambridge, 2000. Pour les contributions signalées du congrès LEST, voir L. BOEVE - L. LEISSSEN (éds.), Sacramental Presence in a Postmodern Context: Fundamental Theological Perspectives (BETL, 160), Leuven, 2001, pp. 161198, 199-205.

29. Cfr. D. JANICAUD, Le tournant théologique de la phénoménologie française, Combas, 1991.

30. J.-L. Marion, Dieu sans l'être, Paris, 1982, et Étant donné: essai d'une phénoménologie de la donation, Paris, 1997. Marion prétend qu'il est possible de montrer phénoménologiquement (et pas seulement théologiquement) la priorité de la logique du don sur celle de l'Être. Pour une approche théologique bienveillante de ce point de vie, voir S. VAN DEN BOSSCHE, God verschijnt toch in de immanentie. De fenomenologische neerlegging van de theologie in Jean-Luc Marions "Étant donné», dans R. WELTEN (éd.), God en het denken. Over de filosofie van Jean-Luc Marion (Annalen van de Thijmgenootschap, 88/2), Nijmegen, 2000, pp. 128-153. Pour ma part, je préfere la vision herméneutico-phénoménologique de Derrida ou de Ricœur, qui mettent l'accent sur la position herméneutique non-réductible du penser humain, et donc sur le caractère principiellement irréductible du langage.

31. Cfr. J.-Y. LACOSTE, Expérience et absolu: Questions disputées sur l'humanité de l'homme, Paris, 1994. 
contraire, il affirme expressément qu'elle est de nature purement phénoménologique. L'élargissement de la phénoménologie est, à ses yeux, une correction et une redéfinition fondamentale de celle-ci.

Le point de départ de ces deux tendances théologico-philosophiques est la déconstruction du partenaire séculier de la théologie moderne de la corrélation, et par là également la faillite du projet de cette théologie de la corrélation. Le contexte actuel est interprété en termes de nihilisme et de déperdition de sens, et il ne peut être sauvé que par une ouverture à une dépendance théologale plus originelle, dans le premier cas une dépendance à laquelle nous participons et, pour le deuxième groupe, un êtreplacé originel dans une position-de-réponse. Dans ces formes de théologie, il n'y a donc pas non plus d'analyse du contexte contemporain en termes de pluralité, à moins que ce ne soit dans le sens du relativisme, du chaos et de l'absence de perspective. Le principe de continuité, mis en avant par la théologie de la corrélation, entre sécularité et tradition est remplacé par un strict principe de discontinuité. Le principe théologique se trouve en porte-à-faux par rapport à l'humain moderne et le fait éclater.

\section{Réformer la théologie moderne de la corrélation}

Le partenaire de la théologie de la corrélation a effectivement été déconstruit, si on entend par là qu'un certain nombre de ses présupposés modernes ont été minés de manière post-moderne. Le ralentissement de la détraditionalisation visible, à cause de la position majoritaire que les Églises chrétiennes maintenaient encore, est également rattrapé $e^{32}$. Penser culture moderne et société en termes de continuité, ou même de consensus, n'apporte plus grand' chose et, d'un point de vue pastoral, ne fonctionne plus. La méthode moderne de corrélation semble être aujourd'hui

32. Les titres des trois comptes-rendus de l'enquête européenne sur les valeurs, en ce qui concerne la Belgique (publiés par, entre autres, Jan Kerkhofs et Karel Dobbelaere) en disent long à ce propos. Le premier, rapportant la recherche effectuée en 1981, est intitulé De stille ommekeer: oude en nieuwe waarden in het België van de jaren tachtig, Tielt, 1984 ( Le changement silencieux: anciennes et nouvelles valeurs dans la Belgique des années quatre-vingts»)). Dans ce volume, la détraditionalisation furtive est démontrée par des chiffres. La Belgique s'est lentement éloignée de son caractère catholique romain classique. Le titre du deuxième volume indique une position plus claire: De versnelde ommekeer: de waarden van de Vlamingen, Walen en Brusselaars in de jaren negentig, Tielt, 1992 («Le changement accéléré: les valeurs des Flamands, des Wallons et des Bruxellois dans les années nonante»). La Belgique se détraditionalisait plus rapidement que jamais. Le titre du troisième livre, Verloren zekerheid: de Belgen en hun waarden, overtuigingen en houdingen, Tielt, 2000 («Certitude perdue: les Belges et leurs valeurs, convictions et comportements»), indique que le processus de détraditionalisation approche de sa réalisation. une stratégie théologique contre-productive, tant d'un point de vue systématique que pastoral. En partant de l'humain-en-général, il devient très difficile de pousser jusqu'au particulier chrétien, si celui-ci n'est pas déjà identifié au premier. Premièrement apparaît en effet la question à propos de ce qu'est cet «humain-en-général». Dans les sciences (humaines) également règne une pluralité méthodologique, dont les résultats ne sont pas toujours complémentaires. Là où elles deviennent normatives, de nombreux présupposés sous-jacents et des intérêts, camouflés ou non, semblent assurément présents. Deuxièmement, à partir d'une analyse de la pluralité radicale, le premier niveau non-réductible pour la réflexion est la particularité elle-même, la narrativité spécifique d'une position existentielle - c'est-à-dire le niveau du récit.

Je l'ai déjà rappelé quelques fois. Une théologie du contexte postmoderne, elle non plus, ne renonce pas au dialogue avec le contexte, et notamment avec les sciences humaines. En même temps, comme Schillebeeckx l'indique lui-même, le théologien se doit «d'être particulièrement critique d'un point de vue herméneutique et, en outre, d'avoir un regard critique sur ce qui, dans le discours ethno-anthropologique au sujet de l'héritage sacramentel spécifiquement chrétien, a été en fait retravaillé à partir de conceptions théologiques» ${ }^{33}$. Cependant, la théologie doit apprendre à ne pas remettre à plus tard la dimension théologale, dans l'espoir de pouvoir l'atteindre, pour ainsi dire automatiquement, à travers les sciences humaines. Celui qui, aujourd'hui, espère encore trouver une telle continuité sera déçu. La conscience de la particularité propre de l'option chrétienne de foi place au premier plan, tant anthropologiquement que théologiquement, l'option de foi du chrétien, la profession de l'engagement de Dieu en faveur de l'homme, en Jésus-Christ, non pas en opposition à l'être-homme du chrétien, mais comme qualification nonréductible de cet être-homme: nous ne vivons pas de structures humaines générales, mais de particularité (dans laquelle ces structures peuvent éventuellement être reconnues a posteriori) ${ }^{34}$. Exprimé de façon lapidaire, cela signifie que ce n'est pas en tant qu'hommes que nous sommes chrétiens, mais en tant que chrétiens que nous sommes hommes, déterminés de façon non-réductible à partir de notre narrativité propre (tout comme d'autres sont hommes dans leur particularité non-réductible). Il s'agit alors de développer des modèles réflexifs permettant de penser

33. SCHILLEBEECKX, Naar een herontdekking, p. 175.

34. À mon sens, ceci est la raison pour laquelle, dans l'analyse de Paul Post (cfr. la note 13), un deuxième secteur se dessine dans le champ des Ritual Studies, qui se démarque quelque peu du premier, et étudie la réalité à partir de cas concrets. Voir également, à ce propos: Hans GEYBELS, Algemeen menselijk of eigen christelijk?. Rituelen en de identiteit van religies, dans Tijdschrift voor Theologie 41 (2001) 221-230. 
également cette qualification. Et précisément pour cela, le dialogue avec la contexte contemporain peut aussi se relever fructueux.

Le modèle de la recontextualisation, aussi bien comme catégorie descriptive que comme catégorie normative, peut être considéré comme la radicalisation (la recontextualisation) de la théologie moderne de la corrélation $^{35}$, précisément parce que, à côté de la notion de continuité, celles de contingence, de particularité, d'altérité et de discontinuité y trouvent surtout une place. Une herméneutique radicalisée en est le fondement. En principe, on pourrait parler d'une «corrélation postmoderne», pour indiquer la continuité avec la théologie moderne, là où celle-ci a ancré méthodologiquement le dialogue avec le contexte. Mais il est préférable d'éviter ce terme. La corrélation respire en effet toujours l'esprit de continuité, d'harmonie et de synthèse. La co-relation désigne le fait que deux partenaires entrent en relation, ou se trouvent en relation l'un avec l'autre (même si celle-ci est réciproquement critique). Dans le contexte post-moderne, par contre, la particularité du récit chrétien (au milieu de la multiplicité des récits) est devenu le point de départ nonréductible de la relation ( $a d$ extra), tandis que, en même temps, la confrontation avec la multiplicité (des autres particularités) fait fonctionner le processus de recontextualisation ad intra. D'où le fait que nous préférons le terme «recontextualisation».

L'expression «tout ce qui vient d'en haut, vient d'en bas» (Kuitert), qui convient très bien à la façon de raisonner de Schillebeeckx, telle que nous l'avons montrée, s'entend mieux, à partir de notre optique, comme suit: «tout ce qui se rapporte à ce qui vient d'en haut, vient d'en bas»: ou bien: «toute parole portant sur ce qui est en haut, vient d'en bas». Cela dit, comment penser cet «en haut» en considération du «en bas»? Nous proposons: en termes d'interruption.

\section{Vers une théologie de l'interruption - entre continuité et discon-} tinuité

Maintenir la perspective de continuité dans notre contexte ressemble à l'accomplissement de la sécularisation moderne, c'est-à-dire à la

35. Dans deux articles, je développe ceci à travers Küng et Schillebeeckx, c'est-à-dire respectivement dans: Kan traditie veranderen? Theologie in het postmoderne tijdgewricht, dans Collationes 26 (1996) 365-385, en référence à: H. KüNG, Theologie im Aufbruch. Eine ökumenische Grundlegung, Munich/Zürich, 1987, reposant sur IDEM - D. TRACY (éds.), Paradigm Change in Theology. A Symposium for the Future, Edinbourg, 1989; et dans Erfgenaam en erflater. Kerkelijke tradities binnen de Traditie, dans $\mathrm{H}$. LOMBAERTS - L. BOEVE (éds.), Traditie en initiatie. Perspectieven voor de toekomst (Nikè, 36), Leuven, 1996, pp. 43-77, en référence à E. SCHILLEBEECKX, Mensen als verhaal van God, Baarn, 1989. sécularisation du caractère de révélation de la foi chrétienne, au nom du religieux humain en général. La révélation ne peut alors être pensée le plus souvent que comme redoublement de ce qui existe déjà à un niveau humain général, ou souvent aussi comme un remplissage narratif de ce dernier. Mais, théologiquement, cela ne tient pas debout. La révélation, pour le chrétien, désigne avant tout l'être-situé de celui-ci dans un engagement envers le Dieu qui s'est fait connaître historiquement en JésusChrist. Cela implique le fait de se situer dans une relation de réponse par rapport à Dieu, le fait de se rapporter à une altérité qui ne saurait être réduite au «propre» (mais qui, notons le, ne peut pas plus être approché ou rencontré sans le propre). La théologie commence ici; le dialogue théologique a pour tâche de clarifier, de refléter cela.

L'hypothèse de recherche à laquelle je donne moi-même la préférence est celle d'un rapport entre des schèmes de pensée ayant trait à la discontinuité et d'autres se rapportant à la continuité, avec la conviction que le fait de donner une priorité trop grande à l'une ou à l'autre est improductif d'un point de vue théologique et pastoral. La catégorie de l'interruption ${ }^{36}$ peut être ici de quelque utilité: interruption ne signifie en effet pas simplement rupture, discontinuité par opposition à toute forme de continuité, mais présuppose une continuité. L'interruption est toujours interruption de quelque chose qui continue après l'interruption. Cependant, dans l'interruption, cette continuation est en même temps interrogée, influencée, transformée, approfondie, enrichie. L'interruption indique les frontières de la continuité et met celle-ci en perspective. L'interruption se produit là où discontinuité et continuité se touchent, aucune des deux n'advenant sans l'autre.

Cette piste permet de poser quelques jalons pour une réflexion subséquente. Nous reprenons l'article de Schillebeeckx comme point de départ. Dans les rites sacramentels d'existence, on peut, à côté d'une double continuité, distinguer également une double discontinuité. Voyons d'abord ce qui concerne la continuité. (1) La première forme de continuité se trouve dans le fondement anthropologique de la praxis rituelle chrétienne, dans l'aspect «rite d'existence» qui peut être discerné dans chaque sacrement. En ce sens, la praxis sacramentelle chrétienne répond à ce que l'on appelle, au sens humain général du terme, la ritualisation. (2) À côté de cette dimension de continuité anthropologique, et à l'intérieur' de celle-ci, on peut également discerner une continuité narrative chrétienne, théologique. Les sacrements sont complètement

36. Pour un déploiement alternatif de ce concept, voir mon article: God onderbreekt de tijd. Over de apocalyptiek als onontkoombare actuele theologische denkfiguur, dans $\mathrm{L}$ BOEVE - J. HAERS (éds.), God ondergronds. Opstellen voor een theologisch vrijdenker. Fs Georges De Schrijver, Averbode, 2001, pp. 377-399. Une version en anglais est publiée dans Louvain Studies 26 (2001) 195-216. 
enracinés dans la particularité chrétienne; ils n'en sont pas seulement l'expression, mais ils sont également constitutifs de cette dernière. Ils parlent d'un Dieu qui s'est révélé dans l'histoire des hommes, et le plus profondément dans l'homme Jésus, confessé par ses disciples comme étant le Christ. En tant que rites situés, ils incarnent la constitution de sens qui se produit à travers le récit chrétien, et ils donnent forme à l'espace symbolique spécifiquement chrétien.

Cette double continuité doit être interrompue, sans quoi la narration chrétienne devient (1) un simple redoublement ou un remplissage du niveau anthropologique. (2) Ou bien, elle se réduit à être de la narrativité pour la narrativité, un récit fermé, dans lequel la donation de sens se trouve enfermée à l'intérieur des frontières de l'horizon herméneutique ${ }^{37}$. La discontinuité est déjà présente au niveau anthropologique, là où les rites interrompent et transforment la vie de tous les jours (une fête, une promotion doctorale, etc. $)^{38}$. Mais la double discontinuité que nous voulons indiquer ici est pensée d'abord à partir de la narrativité chrétienne, et est par conséquent (et simultanément) de nature théologale. Elle se trouve développée à partir des perspectives extérieure et intérieure ou, en d'autres termes, au niveau du rapport entre l'humain en général et le théologique, et à l'intérieur du discours théologique lui-même. La sacramentalité de la praxis liturgico-rituelle des communautés chrétiennes de foi se situe ici.

(1) Premièrement, le narratif chrétien interrompt les structures humaines générales que 1'on peut discerner en elle, et ce à partir du primat de la particularité. Ceci peut être démontré le plus facilement à partir de la perspective extérieure. Là où le narratif chrétien indique les structures humaines générales, là se trouve souvent précisément la différence entre les diverses conceptions de la vie et positions religieuses. Pour exprimer cette idée de façon concise, on pourrait dire: «ce que différentes religions et différentes conceptions de la vie ont en commun les fait se différencier les unes des autres»; ou encore: «ce qui les relie, les distingue». Schillebeeckx, par exemple, découvre les sources de la solennité de Pâques dans un fête agraire du printemps ${ }^{39}$ - ceci peut être vrai, mais, en tant que fête chrétienne du printemps, Pâques se distingue en même temps fortement de telles fêtes, car elle est le fruit d'une transformation résultant d'une perception historique interrompue de façon théologale. L'étude de Pâques comme fête agraire peut ouvrir l'accès à des dimensions précieuses de cette fête chrétienne, et indiquer l'ampleur du rituel festif, mais ne peut en aucun cas renvoyer à la confession rituelle chrétienne de ce que Jésus-

37. Mais, comme nous l'avons dit: il ne peut y avoir de discontinuité sans continuité, ce qui nous mènerait trop facilement vers de l'«escapisme».

38. SCHILLEBEECKX, Naar een herontdekling, p. 183.

39. Ibid., p. 173.
Christ est vivant. C'est sur ce point que la particularité chrétienne donne un tournant irréductible propre à des structures humaines générales. Ici, l'espace herméneutique ouvre une dimension sans laquelle le sacrement ne serait pas sacrement. La même chose peut être dite à propos de la pratique de la prière, des périodes rituelles (comme les temps de jeûne, par exemple), des célébrations communautaires, des rites d'initiation, etc. Ici aussi, on distingue de - on interrompt - ce qui est commun. C'est ce qui se passe, par exemple, pour les trois religions monothéistes, également appelées religions du livre, ou religions historiques de révélation. Aussi bien les conceptions de Dieu qu'elles véhiculent, que la façon dont elles font fonctionner le «livre», que leur façon de mettre en rapport Dieu et histoire, marquent surtout la différence entre ces trois.

(2) Ensuite, d'un point de vue théologal, le narratif chrétien interrompt l'humain-en-général, non pas parce que celui-ci ne serait pas «vrai» ou n'aurait aucune valeur, mais parce que les sacrements chrétiens, pour le chrétien, ne tirent pas leur signification la plus profonde de l'humain-engénéral. C'est parce qu'ils parlent de Dieu que les sacrements sont tels. La sacramentalité est interruption venant de Dieu.

En ce qui concerne cette dimension théologale, Schillebeeckx part de ce qu'il a développé antérieurement à propos de l'expérience chrétienne du contraste: le fait d'être confronté à des traces de Dieu dans l'existence, traces qui sont «purifiées en rite» dans la praxis sacramentelle. En tant qu'expériences de contraste, les traces de Dieu doivent être pensées comme des expériences d'altérité non-maîtrisable, de conflit, d'irréconciliabilité, interprétées sur l'arrière-fond du récit chrétien. Mieux encore: théologiquement parlant, elles constituent la frontière du «récit chrétien» - sa condition de possibilité et sa limite critique. Comme expérience de l'altérité, elles interrompent des récits en cours, également des récits chrétiens, et introduisent la perspective théologale. Dieu n'est alors pas pensé d'abord exclusivement à l'intérieur de récits, mais précisément comme Celui qui échappe précisément toujours déjà aux récits, et dont (ou de l'«échappement» duquel) précisément le récit chrétien témoigne un Dieu qui ne se laisse pas trop rapidement réconcilier, dont la trace introduit précisément l'irréconciliabilité. De ce point de vue, la recontextualisation n'est pas un procédé purement anthropologique propre aux traditions et aux récits chrétiens, mais précisément le produit du rapport au Dieu qui alimente nos récits, les interrompt, mais qui ne peut être capturé en eux. Tout ceci s'applique à notre praxis sacramentelle, ainsi qu'à la réflexion à son sujet (par exemple à propos de la présence sacramentelle $\left.{ }^{40}\right)$. Là également, Dieu et la grâce de Dieu ne sont pas des don-

40. À comparer avec L. HeMMING à propos de la doctrine de la transsubstantiation de Thomas d'Aquin (After Heidegger: Transsubstantion, dans BOEVE - LEIJSSEN (éds.), 
nées palpables, saisissables, mais elles sont à situer à l'intérieur d'un rapport à un Autre, ouvert à l'intérieur de l'espace herméneutique propre du récit chrétien, mais qui ne peut être réduit à ce dernier ${ }^{41}$.

En tant qu'incarnations de significations profondes, les rites chrétiens ancrent le récit chrétien dans la matérialité et la corporéité de notre êtrehomme, et interrompent celles-ci à partir de la confession de foi chrétienne de leur caractère de créature et de l'espérance de leur accomplissement - une confession de foi qui, à son tour, est interrompue par le Dieu qui exprime l'être-Créateur et l'être-Rédempteur.

\section{Conclusion}

Je suis conscient de ne pas donner, à partir de ces considérations, de réponse toute prête à la problématique pastorale et théologique que recèle le thème de cette rencontre, à savoir le rapport entre les rites d'existence et les sacrements.

Par contre, j'espère (1) avoir donné des indications concernant les questions de théologie fondamentale qui peuvent surgir en lien à cette question, (2) avoir mis en lumière l'importance relative de l'étude anthropologique et (humano-) scientifique pour la théologie, (3), avoir indiqué les limites de la théologie moderne de la corrélation pour une pratique théologique en contexte post-moderne, (4) et cela parce que ce contexte n'est plus analysable avant tout (seulement) en termes de sécularité, mais plutôt en termes de particularité et de pluralité. Toutes ces considérations soutiennent (et s'appuient sur) mon hypothèse de recherche selon laquelle la réflexion théologique actuelle à propos des rites et des sacrements ne peut nier l'importance de la recherche anthropologique (ou de l'ensemble que l'on nomme aujourd'hui les Ritual Studies), mais que, en même temps (et plus qu'autrefois), elle doit être explicitement attentive à la spécificité chrétienne de la praxis sacramentelle chrétienne. (5) Je pense également avoir indiqué que ceci ne signifie pas un retrait de la théologie hors du dialogue interdisciplinaire (et interreligieux), mais est précisément le fruit de ce dialogue.

Ce mouvement m'a obligé à tracer quelques lignes d'une théologie de l'interruption, en vertu de laquelle la continuité est reliée à la

Sacramental Presence in a Postmodern Context, pp. 293-303). Thomas conserve la physique d'Aristote, mais l'interrompt en même temps, la met en perspective.

41. Voir, à ce propos, la contribution de L.-M. CHAUVET, The Broken Bread as Theological Figure of Eucharistic Presence, dans BOEVE - LEIJSSEN (éds.), Sacramental Presence in a Postmodern Context, pp. 230-256; ausssi apparue comme Le pain rompu comme figure théologique de la présence eucharistique, dans ID. (éd.), Contemporary Sacramental Contours of a God Incarnate (Textes et études liturgiques / Studies in Liturgy, 16), Leuven, 2001, pp. 14-38. discontinuité, et inversement. (6) Dans la perspective selon laquelle «ce qui nous lie nous distingue», la sacramentalité devient avant tout indication de la particularité spécifique de la praxis rituelle chrétienne. (7) Deuxièmement, la sacramentalité évoque la dimension théologale de cette praxis, dimension qui peut être pensée comme interruption (de cette dernière).

Platte-Lostraat 592

B-3010 Kessel-Lo
Lieven BOEVE 